\title{
A Fifth-Order Korteweg-de Vries Equation for Shallow Water with Surface Tension: Multiple Soliton Solutions
}

\author{
A.M. WAZWAZ* \\ Department of Mathematics, Saint Xavier University, Chicago, IL 60655, USA
}

(Received August 7, 2015; in final form March 3, 2016)

\begin{abstract}
In this work we study a fifth-order Korteweg-de Vries equation for shallow water with surface tension derived by Dullin et al. The fifth-order Korteweg-de Vries equation, derived by using the nonlinear/non-local transformations introduced by Kodama, and the Camassa-Holm equation with linear dispersion, have very different behaviors despite being asymptotically equivalent. We use the simplified form of the Hirota direct method to derive multiple soliton solutions for this equation.
\end{abstract}

DOI: 10.12693/APhysPolA.130.679

PACS/topics: 02.70.Wz, 02.30.Lk, 02.30.Jr

\section{Introduction}

In the context of nonlinear fifth-order KdV type equations, studies are flourishing because these equations are able to describe the real features in a variety of scientific applications and engineering areas and would have much practical/physical meaning [1-17]. Fifthorder $\mathrm{KdV}$ (KdV5) type equations take the form

$$
u_{t}=u_{x x x x x}+f\left(x, t, u_{x}, u_{x x}, u_{x x x}\right) \text {, }
$$

arising naturally in modeling many different wave phenomena such as gravity-capillary waves, the propagation of shallow water waves over a flat surface and magnetosound propagation in plasmas [5]. The fifth-order KdV type equations appear in the literature in three main families, given as:

1. The first family reads [18]:

$$
u_{t}+a u u_{x}+b u_{3 x}-r u_{5 x}=0 \text {, }
$$

where $a, b, r$ are constants. This equation is called the Kawahara equation which models the dynamics of long waves in a viscous fluid.

2. The second family of nonlinear fifth-order $\mathrm{KdV}$ $(\mathrm{fKdV})$ equations in its standard form reads [18-30]:

$$
u_{t}+\alpha u^{2} u_{x}+\beta u_{x} u_{x x}+\gamma u u_{3 x}+u_{5 x}=0,
$$

where $\alpha, \beta$, and $\gamma$ are arbitrary nonzero and real parameters, and $u=u(x, t)$ is a sufficiently smooth function. The fifth-order KdV Eqs. (3) involve the linear dispersive term $u_{5 x}$ in addition to three nonlinear terms. Because the parameters $\alpha, \beta$, and $\gamma$ are arbitrary and take different values, this will drastically change the characteristics of the fKdV Eq. (3). A variety of the fKdV equations can be developed by changing the real values of the parameters $\alpha, \beta$, and $\gamma[18]$. The most wellknown fifth-order KdV equations which were examined thoroughly are the Sawada-Kotera (SK) equation, the Caudrey-Dodd-Gibbon equation, the Lax equation, the

\footnotetext{
*e-mail: wazwaz@sxu.edu
}

Kaup-Kuperschmidt (KP) equation, and the Ito equation. These forms are:

(i) the Sawada-Kotera (SK) equation [18] is given by

$$
u_{t}+5 u^{2} u_{x}+5 u_{x} u_{x x}+5 u u_{3 x}+u_{5 x}=0
$$

(ii) the Caudrey-Dodd-Gibbon equation (CDG) [18] is given by

$$
u_{t}+180 u^{2} u_{x}+30 u_{x} u_{x x}+30 u u_{x x x}+u_{x x x x x}=0
$$

(iii) the Lax equation [18] reads

$$
u_{t}+30 u^{2} u_{x}+20 u_{x} u_{x x}+10 u u_{3 x}+u_{5 x}=0 ;
$$

(iv) the Kaup-Kuperschmidt (KK) equation [18] reads

$$
u_{t}+20 u^{2} u_{x}+25 u_{x} u_{x x}+10 u u_{3 x}+u_{5 x}=0
$$

and is characterized by

$$
\beta=\frac{5}{2} \gamma, \alpha=\frac{1}{5} \gamma^{2}
$$

(v) the Ito equation [18]

$$
u_{t}+2 u^{2} u_{x}+6 u_{x} u_{x x}+3 u u_{3 x}+u_{5 x}=0 .
$$

It was found that the SK, CDG, Lax, and KK equations belong to the completely integrable hierarchy of higherorder KdV equations. These four equations have infinite sets of conservation laws, and therefore these equations have $N$-soliton solutions. However, the Ito equation is not completely integrable but has a limited number of special conservation laws.

3. The third family reads [19-30]:

$$
u_{t t t}-u_{t x x x x}-\alpha\left(u_{t} u_{x}\right)_{x x}-\beta\left(u_{x} u_{x t}\right)_{x}=0,
$$

or equivalently

$$
u_{t t t}-u_{t x x x x}-\alpha\left(u_{t} u_{x}\right)_{x x}-\beta\left(u_{x} u_{x x}\right)_{t}=0 .
$$

The third family is integrable only if $\alpha=\beta$.

However, Dullin et al. $[1,2]$ used the Kodama transformation given in $[3,4]$ to transform the Camassa-Holm $(\mathrm{CH})$ equation to a fifth-order $\mathrm{KdV}$ equation, referred to by KdV5, given by

$$
\begin{gathered}
u_{t}+c u_{x}+3 u u_{x}+5 \alpha^{2}\left(u u_{x x x}+2 u_{x} u_{x x}\right) \\
+\frac{15}{2} \frac{\alpha^{2}}{\beta} u^{2} u_{x}+\beta\left(\alpha^{2} u_{x x x x x}+u_{x x x}\right)=0,
\end{gathered}
$$

which works as a model for the shallow water waves with 
surface tension, $u(x, t)$ is the fluid velocity, $\alpha^{2}$ and $\beta$ are the length scales, and $c$ is the linear wave speed [1-6]. Unlike the second family of fifth-order $\mathrm{KdV}$ equations, the KdV5 includes two linear dispersive terms $u_{x x x}$ and $u_{x x x x x}$. For $\alpha=0$, the KdV5 (12) equation recovers the standard $\mathrm{KdV}$ equation.

Through the Kodama transformation [1-6]:

$$
U=u+\xi\left(\frac{\alpha^{2}}{\beta} u^{2}+2 \frac{\alpha^{2}}{\beta} u_{x} \partial^{-1} u\right)+2 \delta^{2} \alpha^{2} u_{x x},
$$

the $\mathrm{CH}$ equation [9]

$$
m_{t}+c m_{x}+U m_{x}+2 m U_{x}+\beta U_{x x x}=0
$$

can be transformed to the KdV5 Eq. (12), where $m=$ $U^{2}-\alpha^{2} U$ is a momentum variable, and $U(x, t)$ represents the height of the water free surface above a flat bottom. The $\mathrm{CH}$ equation can be derived as an asymptotic model for long gravity waves at the surface of shallow water. The $\mathrm{CH}$ equation retains non dominant terms with respect to the dominant balance between nonlinearity and dispersion modeled by the $\mathrm{KdV}$ equation. Besides being a model equation for water waves, the $\mathrm{CH}$ equation has its integrable bi-Hamiltonian structure, and has peaked solitary wave solutions [9].

In Refs. [1, 2], it was shown that the asymptotic equivalence of the $\mathrm{CH}$ equation to the $\mathrm{KdV} 5$ equation breaks down in the limit $\beta \rightarrow 0$, because the transformation as well as the resulting equation contain terms divided by $\beta$, and the $\mathrm{CH}$ equation provides peak on solutions whereas the KdV5 equation gives soliton solutions as will be discussed later. Moreover, the $\mathrm{CH}$ equation does not contain the term $u_{x x x x x}$ that makes it easier to integrate numerically if compared with the KdV5 Eq. (12).

In Refs. [1, 2], it was shown that the $\mathrm{CH}$ equation is asymptotically equivalent to KdV5 equation by using the nonlinear/ nonlocal transformations introduced in the Kodama transformation [3, 4]. Moreover, Dullin et al. $[1,2]$ classified the travelling wave solutions of the $\mathrm{KdV}$ equation as a function of the Bond number by using phase plane analysis. In Ref. [5], the authors examined the binary Bell polynomials, Lax pair and the infinite conservation laws for the KdV5 equation.

It is interesting to note that the extended $\mathrm{KdV}$ equation, as first called by Marchant and Smyth [31], of second order in small parameters, was subjected to useful studies in [32-34] and some of the references therein. In Ref. [31], it is shown that the extended KdV equation can be transformed (to its order of approximation) to a higher-order member of the KdV hierarchy of integrable equations. However, in Ref. [32], the equations obtained in the higher orders of approximation, in general, also differ from the $\mathrm{KdV}$ equation with higher order corrections. Also, including surface tension in general does not alter the structure of the leading order and higher order equations [32]. Moreover, in [33], the derivation of a KdV type equation, second order in small parameters, containing terms from the bottom function was examined. The energy invariant for shallow-water waves and the Korteweg-de Vries equation were examined in detail in [34]. Generally, KdV2 equation (second order with respect to small parameters) are examined thoroughly in $[31-34]$ in addition to $[1,2]$.

Studies of finding soliton solutions of the nonlinear equations attracted huge number of works in a variety of fields in [19-30] and some of the references therein. Towards this goal, a variety of powerful methods to construct multiple soliton solutions has been established in the fields of mathematical physics and engineering. Examples of the methods that have been used are the Hirota bilinear method [7-30], the simplified Hirota method [8], the Bäcklund transformation method, the Darboux transformation, Pfaffian technique, the inverse scattering method, the Painlevé analysis, the generalized symmetry method, the subsidiary ordinary differential equation method, the coupled amplitude-phase formulation, the sine-cosine method, the sech-tanh method, the mapping and the deformation approach, and many other methods. The Hirota bilinear method [7], and the simplified Hirota method developed in [8] are rather heuristic and significant to handle equations with constant coefficients. These two methods possess powerful features that make it practical for the determination of single soliton and multiple soliton solutions for a wide class of nonlinear evolution equations. The simplified Hirota method [8] does not depend on the construction of the bilinear forms, instead it assumes that the multi-soliton solutions can be expressed as polynomials of exponential functions. The computer symbolic systems such as Maple and Mathematica allow us to perform complicated and tedious calculations.

In this work we plan to use the simplified Hirota method to formally derive multiple soliton solutions for the KdV5 Eq. (12). The multiple singular soliton solutions will be examined as well.

\section{The KdV5 equation}

In this section we will study the $\mathrm{KdV} 5$ equation

$$
\begin{aligned}
& u_{t}+c u_{x}+3 u u_{x}+5 \alpha^{2}\left(u u_{x x x}+2 u_{x} u_{x x}\right) \\
& +\frac{15}{2} \frac{\alpha^{2}}{\beta} u^{2} u_{x}+\beta\left(\alpha^{2} u_{x x x x x}+u_{x x x}\right)=0, \beta \neq 0 .
\end{aligned}
$$

To determine the dispersion relation for (15) we substitute

$$
u(x, t)=\mathrm{e}^{\theta_{i}}, \theta_{i}=k_{i} x-c_{i} t,
$$

into the linear terms of (15) and solve the resulting equation for the dispersion relation $c_{i}$ to find that

$$
c_{i}=c k_{i}+\beta\left(k_{i}^{3}+\alpha^{2} k_{i}^{5}\right), i=1,2,3 .
$$

Consequently, the phase variables read

$$
\theta_{i}=k_{i} x-\left[c k_{i}+\beta\left(k_{i}^{3}+\alpha^{2} k_{i}^{5}\right)\right] t, i=1,2,3 .
$$

To determine the single soliton solution, we use the transformation

$$
u(x, t)=R(\ln f(x))_{x x},
$$

where the auxiliary function $f(x, t)$, for the single soliton solution is given by 
$f(x, t)=1+\mathrm{e}^{\theta_{1}}=1+\mathrm{e}^{k_{1} x-\left(c k_{1}+\beta\left(k_{1}^{3}+\alpha^{2} k_{1}^{5}\right)\right) t}$.

Substituting (20) into (15) and solving for $R$ we find

$R=4 \beta$.

This in turn gives the single soliton solution as

$$
u(x, t)=\frac{4 \beta k_{1}^{2} \mathrm{e}^{k_{1} x-\left[c k_{1}+\beta\left(k_{1}^{3}+\alpha^{2} k_{1}^{5}\right)\right] t}}{\left\{1+\mathrm{e}^{k_{1} x-\left[c k_{1}+\beta\left(k_{1}^{3}+\alpha^{2} k_{1}^{5}\right)\right] t}\right\}^{2}} .
$$

Notice that using the auxiliary function as

$$
f(x, t)=1-\mathrm{e}^{\theta_{1}}=1-\mathrm{e}^{k_{1} x-\left[c k_{1}+\beta\left(k_{1}^{3}+\alpha^{2} k_{1}^{5}\right)\right] t},
$$

gives the single singular soliton solution

$$
u(x, t)=-\frac{4 \beta k_{1}^{2} \mathrm{e}^{k_{1} x-\left[c k_{1}+\beta\left(k_{1}^{3}+\alpha^{2} k_{1}^{5}\right)\right] t}}{\left\{1-\mathrm{e}^{k_{1} x-\left[c k_{1}+\beta\left(k_{1}^{3}+\alpha^{2} k_{1}^{5}\right)\right] t}\right\}^{2}},
$$

which blows up when the denominator becomes zero for specific values of $x, t, \alpha^{2}, \beta$ and $k_{1}$. For the two soliton solutions we set the auxiliary function as

$$
f(x, t)=1+\mathrm{e}^{\theta_{1}}+\mathrm{e}^{\theta_{2}}+a_{12} \mathrm{e}^{\theta_{1}+\theta_{2}},
$$

where the phase variables $\theta_{i}, i=1,2,3$ are given earlier in (18), and $a_{12}$ is the phase shift that will be determined. Substituting (25) and (19) into (15) and solving for the phase shift $a_{12}$, we find

$$
a_{12}=\frac{\left(k_{1}-k_{2}\right)^{2}}{\left(k_{1}+k_{2}\right)^{2}},
$$

which can be generalized to

$$
a_{i j}=\frac{\left(k_{i}-k_{j}\right)^{2}}{\left(k_{i}+k_{j}\right)^{2}}, 1 \leq i<j \leq 3 .
$$

Substituting (25),(26) into (19) gives the two soliton solutions.

However, using the auxiliary functions as

$$
f(x, t)=1-\mathrm{e}^{\theta_{1}}-\mathrm{e}^{\theta_{2}}+a_{12} \theta_{1}+\theta_{2},
$$

gives the two singular soliton solutions.

For the three soliton solutions, we set the auxiliary function by

$$
\begin{aligned}
& f(x, t)=1+\mathrm{e}^{\theta_{1}}+\mathrm{e}^{\theta_{2}}+\mathrm{e}^{\theta_{3}}+a_{12} \mathrm{e}^{\theta_{1}+\theta_{2}}+a_{13} \mathrm{e}^{\theta_{1}+\theta_{3}} \\
& +a_{23} \mathrm{e}^{\theta_{2}+\theta_{3}}+b_{123} \mathrm{e}^{\theta_{1}+\theta_{2}+\theta_{3}} .
\end{aligned}
$$

Proceeding as before, we find

$$
b_{123}=a_{12} a_{23} a_{13} .
$$

The three soliton solutions are obtained by substituting (29) into (19). This shows that the KdV5 equation (15) gives $N$-soliton solutions for finite $N$, where $N \geq 1$.

Moreover, using the auxiliary function in the form

$$
\begin{gathered}
f(x, t)=1-\mathrm{e}^{\theta_{1}}-\mathrm{e}^{\theta_{2}}-\mathrm{e}^{\theta_{3}}+a_{12} \mathrm{e}^{\theta_{1}+\theta_{2}}+a_{13} \mathrm{e}^{\theta_{1}+\theta_{3}} \\
+a_{23} \mathrm{e}^{\theta_{2}+\theta_{3}}-a_{12} a_{13} a_{23} \mathrm{e}^{\theta_{1}+\theta_{2}+\theta_{3}},
\end{gathered}
$$

gives three singular soliton solutions.

\section{Discussion}

In this work we studied the KdV5 equation which works as a model for the shallow water waves with surface tension. The KdV5 equation and the $\mathrm{CH}$ equation with linear dispersion, have very different behaviors despite being asymptotically equivalent. We should that the KdV5 equation gives multiple soliton solutions, whereas the Camassa-Holm equation provides peak on solutions. We used the simplified Hirota method to formally derive the multiple soliton solutions for the KdV5 equation.

\section{References}

[1] H.R. Dullin, G.A. Gottwald, D.D. Holm, Fluid Dyn. Res. 33, 73 (2003).

[2] H.R. Dullin, G.A. Gottwald, D.D. Holm, Physica D 190, 1 (2004).

[3] Y. Kodama, Phys. Lett. A 107, 245 (1985).

[4] Y. Kodama, Phys. Lett. A 123, 276 (1987).

[5] W.-R. Sun, W.-R. Shan, Y. Jiang, P. Wang, B. Tian, Eur. Phys. J. D 69, 47 (2015).

[6] N. Khanal, R. Sharma, J. Wu, J.-M. Yuan, Discr. Contin. Dyn. Syst. 12, 442 (2009).

[7] R. Hirota, The Direct Method in Soliton Theory, Cambridge University Press, Cambridge 2004.

[8] W. Hereman, A. Nuseir, Math. Comput. Simulat. 43, 13 (1997).

[9] R. Camassa, D. Holm, Phys. Rev. Lett. 71, 1661 (1993).

[10] H. Leblond, D. Mihalache, Phys. Rep. 523, 61 (2013).

[11] H. Leblond, D. Mihalache, Phys. Rev. A 79, 063835 (2009).

[12] C.M. Khalique, J. Appl. Math. 2013, 1 (2013).

[13] C.M. Khalique, K.R. Adem, Math. Comput. Modell. 54, 184 (2011).

[14] X. Lu, W.-X. Ma, C.M. Khalique, Appl. Math. Lett. 50, 37 (2015).

[15] W.X. Ma, Z. Zhu, Appl. Math. Computat. 218, 11871 (2012)

[16] W.X. Ma, A. Abdeljabbar, M.G. Asaad, Appl. Math. Comput. 217, 10016 (2011).

[17] M. Dehghan, A. Shokri, Comput. Math. Simul. 79 , 700 (2008).

[18] A.M. Wazwaz, Partial Differential Equations and Solitary Waves Theorem, Springer and HEP, Berlin 2009.

[19] A.M. Wazwaz, Phys. Scr. 83, 015012 (2011).

[20] A.M. Wazwaz, Phys. Scr. 83, 035003 (2011).

[21] A.M. Wazwaz, Phys. Scr. 84, 025007 (2011).

[22] G.Q. Xu, A.M. Wazwaz, Math. Meth. Appl. Sci. In Press New bilinearization, Bäcklund transformation and infinite conservation laws for the KdV6 equation with binary Bell polynomials, (2016).

[23] G.Q. Xu, S.B. Li, Comput. Phys. Commun. 161, 65 (2004).

[24] G.Q. Xu, Phys. Rev. E 74, 027602 (2006).

[25] G.Q. Xu, Z.Z. Huang, Chin. Phys. Lett. 30, 030202 (2013).

[26] G.Q. Xu, Phys. Scr. 89, 125201 (2014).

[27] A.M. Wazwaz, G.Q. Xu, Commun. Theor. Phys. 63, 727 (2015).

[28] A.M. Wazwaz, Appl. Math. Comput. 190, 633 (2007). 
[29] A.M. Wazwaz, Appl. Math. Comput. 190, 1198 (2007).

[30] A.M. Wazwaz, Commun. Nonlinear Sci. Numer. Simul. 12, 1395 (2007).

[31] T.R. Marchant, N.F. Smyth, IMA J. Appl. Math. 56, 157 (1996)
[32] G.I. Burde, A. Sergyeyev, J. Phys. A Math. Theor. 46, 075501 (2013).

[33] A. Karczewska, P. Rozmej, E. Infeld, Phys. Rev. E 90, 012907 (2014).

[34] A. Karczewska, P. Rozmej, E. Infeld, Phys. Rev. E 92, $053202(2015)$ 